\title{
$\mathrm{HSC} / 00 / 01$
}

\section{Hurst analysis of electricity price dynamics}

\author{
Rafał Weron* \\ Beata Przybyłowicz*
}

* Hugo Steinhaus Center, Wrocław University of Technology, Poland

Hugo Steinhaus Center Wrocław University of Technology Wyb. Wyspiańskiego 27, 50-370 Wrocław, Poland http://www.im.pwr.wroc.pl/ hugo/ 


\title{
Hurst analysis of electricity price dynamics
}

\author{
Rafal Weron ${ }^{1,2}$ and Beata Przybyłowicz \\ Hugo Steinhaus Center for Stochastic Methods, \\ Wrocław University of Technology, 50-370 Wrocław, Poland
}

\begin{abstract}
The price of electricity is extremely volatile, because electric power cannot be economically stored, end user demand is largely weather dependent, and the reliability of the grid is paramount. However, underlying the process of price returns is a strong mean-reverting mechanism. We study this feature of electricity returns by means of Hurst R/S analysis.
\end{abstract}

PACS: 05.45.Tp, 89.30.+f, 89.90.+n

Keywords: Econophysics, electricity price, mean-reversion, Hurst analysis

\section{Introduction}

Standard statistical analysis begins by assuming that the system under study is primarily random, i.e. that the underlying process has many component parts and the interaction of those components is so complex that a deterministic explanation is not possible. In this setup the system is usually modeled by a random walk type process, which implies that the events measured are independent and identically distributed (iid). In other words, the events must not influence one another and they must all be equally likely to occur.

However, there exists a strong evidence that price processes of financial assets do not fall into this category $[1,2,3]$. These processes seem to be persistent with memory lasting up to a few years [4] and possess a non-trivial autocorrelation structure $[5,6]$. Recently it has been suggested [7] that electricity price processes are neither persistent nor random walks, and rather fall into the category of mean-reverting (anti-persistent) processes. In this paper we investigate it more thoroughly by means of Hurst R/S analysis.

\section{Hurst analysis}

In the early 20th century, a celebrated british hydrologist H.E.Hurst worked on the Nile River Dam Project. He had studied the eight and a half century long record that the Egyptians had kept of the Nile River's overflows. The record did not appear to be a white noise. Larger-than-average overflows were more likely to be

\footnotetext{
${ }^{1}$ Corresponding author. E-mail address: rweron@im.pwr.wroc.pl

${ }^{2}$ Research supported by KBN Grant no. 8 T10B 03417.
} 
followed by more large overflows. Abruptly, the process would change to a lowerthan-average overflow, which was followed by other lower-than-average overflows. There appeared to be cycles and long-term memory in the data. However, standard analysis revealed no statistically significant correlations between observations. As a result Hurst developed a method called rescaled range $(\mathrm{R} / \mathrm{S})$ for distinguishing completely random time series from correlated time series $[8,9,10]$.

The analysis begins with dividing a time series (of returns) of length $N$ into $d$ subseries of length $n$. Next for each subseries $m=1, \ldots, d$ : $1^{\circ}$ find the mean $\left(E_{m}\right)$ and standard deviation $\left(S_{m}\right) ; 2^{\circ}$ normalize the data $\left(Z_{i, m}\right)$ by subtracting the sample mean $X_{i, m}=Z_{i, m}-E_{m}$ for $i=1, \ldots, n ; 3^{\circ}$ create a cumulative time series $Y_{i, m}=\sum_{j=1}^{i} X_{j, m}$ for $i=1, \ldots, n ; 4^{\circ}$ find the range $R_{m}=\max \left\{Y_{1, m}, \ldots, Y_{n, m}\right\}-$ $\min \left\{Y_{1, m}, \ldots, Y_{n, m}\right\} ; 5^{\circ}$ rescale the range $R_{m} / S_{m}$. Finally, the mean value of the rescaled range for subseries of length $n$ is $(R / S)_{n}=\frac{1}{d} \sum_{m=1}^{d} R_{m} / S_{m}$.

After the analysis is conducted for all possible divisors of $N$, we can plot the $(R / S)_{n}$ statistics against $n$ on a double-logarithmic paper. If the returns process is white noise then the plot is roughly a straight line with slope 0.5 . If the process is persistent then the slope is greater than 0.5 ; if it is anti-persistent then the slope is less than 0.5. The significance level is usually chosen to be $\sqrt{1 / N}-$ the standard deviation of a Gaussian white noise. However, it should be noted that for small $n$ there is a significant deviation from the 0.5 slope. For this reason the theoretical (i.e. for white noise) values of the $\mathrm{R} / \mathrm{S}$ statistics are approximated by $[3,4]$ (see also [11])

$$
\mathbf{E}(R / S)_{n}= \begin{cases}\frac{n-\frac{1}{2}}{n} \frac{\Gamma\left(\frac{n-1}{2}\right)}{\sqrt{\pi} \Gamma\left(\frac{n}{2}\right)} \sum_{i=1}^{n-1} \sqrt{\frac{n-i}{i}} & \text { for } \quad n \leq 340, \\ \frac{n-\frac{1}{2}}{n} \frac{1}{\sqrt{n \frac{\pi}{2}}} \sum_{i=1}^{n-1} \sqrt{\frac{n-i}{i}} & \text { for } n>340 .\end{cases}
$$

\section{$3 \quad$ Electricity markets}

The deregulation of the electricity industry, which has begun not more than ten years ago, is giving way to a global trend toward the commoditization of electric energy $[12,13]$. This trend has recently intensified in Europe and North America, where market forces have pushed legislators to begin removing artificial barriers that shielded electric utilities from competition.

Organizations which have been used to long-term fixed price contracts are now becoming increasingly exposed to price volatility and, of necessity, are seeking to hedge and speculatively trade to reduce their exposure to price risk. However, we have to bear in mind that electricity markets are not anywhere near as straightforward as financial or even other commodity markets. They have to deal with the added complexity of physical substance, which cannot simply be manufactured, transported and delivered, at the press of a button.

Unlike other commodities, electricity cannot be stored. Therefore, a delicate balance must be maintained between generation and consumption - 24 hours a day, 7 days a week, 8,760 hours a year. Electric power may be generated from natural gas, coal, oil, nuclear fuel, falling water, geothermal steam, alternative resources such as cogeneration, and from renewable resources such as wind power, solar energy and 
biomass. Although the principles of generating electricity are simple, generating electricity for a country or a state the size of California, both in terms of geographic area and population, means a complex balancing process. Certain requirements must be met to ensure system reliability. For example, under deregulation the new California market features "must-run" generation. This is generation that utilities must produce, either to maintain system reliability or to meet requirements imposed by utility regulators. In return for payment, the system operator (ISO) may call upon the owner of a must-run generating unit to run the unit when required for grid reliability.

After electricity is generated, it is transmitted over high-voltage power lines, usually at voltages from 50 to $500 \mathrm{kV}$. Prior to electric industry restructuring, electric utilities transferred power to one another using their transmission system. They also provided service to transmission-service customers, usually large industrial plants. Presently, anyone who owns transmission facilities, or who has firm contractual rights to use transmission facilities to be operated by the ISO, is referred to as a transmission owner. These transmission owners calculate and monitor transmissionline capacities in order to avoid line overloading and possible damage to equipment.

One of the biggest problems faced by the system operator is congestion. It occurs when there is insufficient transfer capacity to simultaneously implement all of the preferred schedules that scheduling coordinators submit to the ISO. The California ISO uses a zone-based approach to manage congestion. A zone is a portion of the ISO-controlled grid within which congestion is expected to occur infrequently or have relatively low congestion-management costs. When congestion occurs, zonal prices supersede power exchange's (PX) market clearing price, which is based on the aggregated energy supply and demand curve intersection point for each hour. In such a case, electricity prices can increase or decrease dramatically. It is even possible for the prices to be negative (see Fig. 1)!

The whole complex process of electricity price formation results in behavior not observed in the financial or even other commodity markets. It is thus extremely interesting to investigate this new world.

\section{Empirical analysis}

The first analyzed time series consists of the California Power Exchange (CalPX) market clearing prices (spot market) for every hour since April 1st, 1998, 0:00 until January 31st, 2000, 24:00. This gives us 16104 data points, which are illustrated in the top panel of Fig. 2. Because the series includes a very strong daily cycle we have created a 671 days long sequence of average daily prices (as in [7]). All further analysis is conducted on the 670 days long sequence of their logarithmic returns, see bottom panel of Fig. 2 .

The first step of the Hurst analysis consists of dividing the sample into subsamples of equal length. Since the number 670 has only four divisors greater or equal to 10 (for subsamples of smaller length errors are too large) we have analyzed a sample of length 660, which has 17 divisors greater or equal to 10: 10, 11, 12, 15, 20, 22, $30,33,44,55,60,66,110,132,165,220$, and 330 . The next step is to calculate 


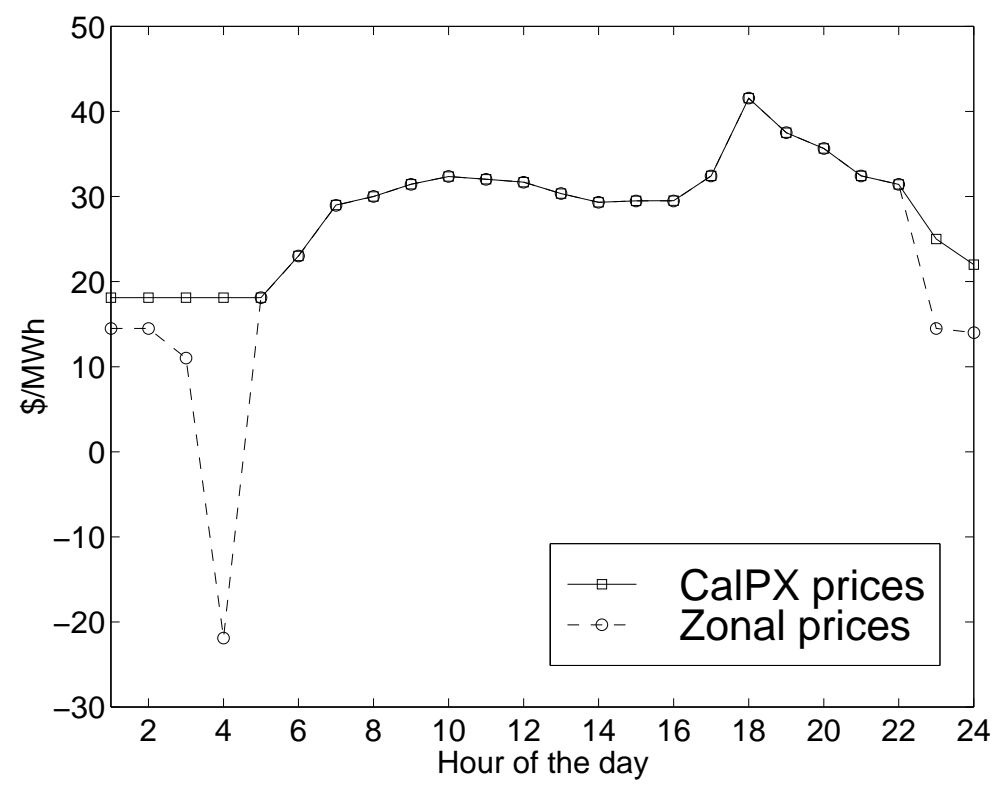

Figure 1: California Power Exchange (CalPX) market clearing prices and Palo Verde zone clearing prices for each hour of November 29th, 1999.
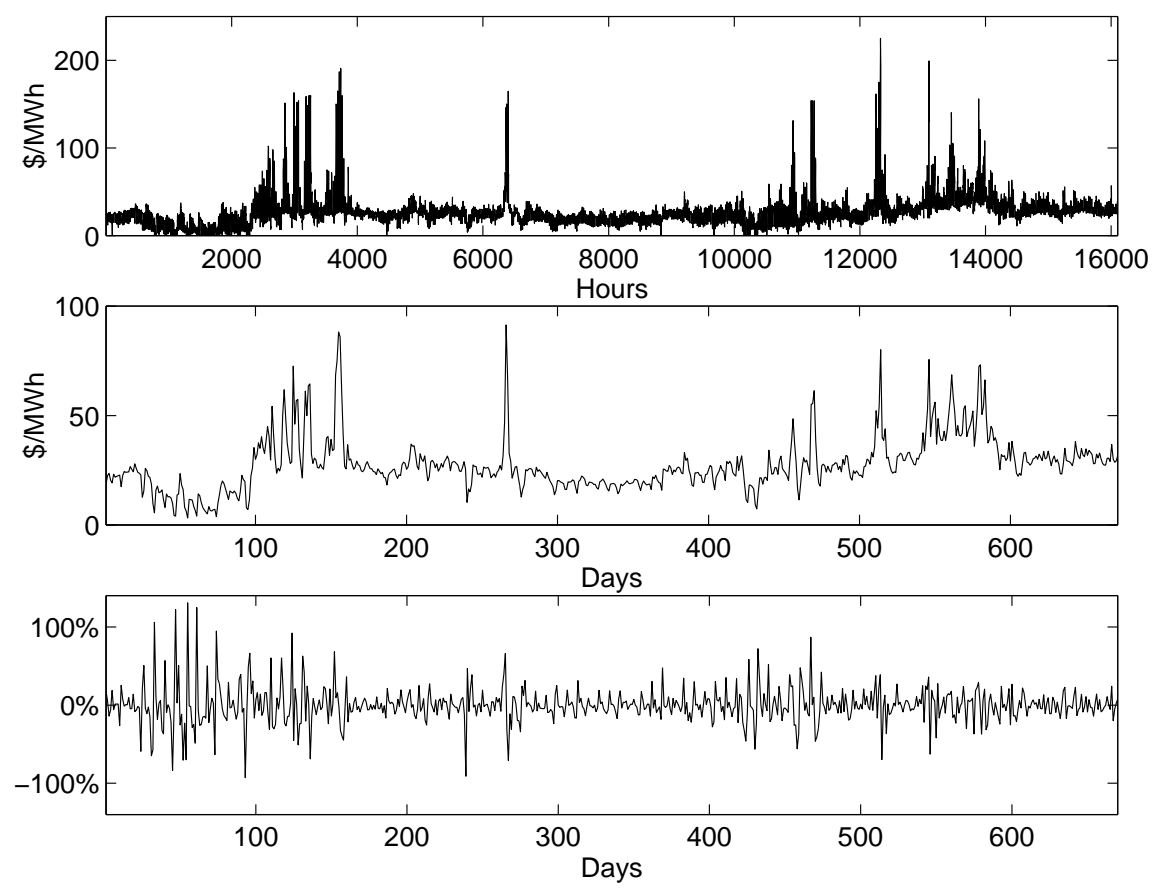

Figure 2: CalPX market clearing prices for every hour since April 1st, 1998, 0:00 until January 31st, 2000, 24:00 (top), average daily prices (middle), and logarithmic returns of average daily prices (bottom). 
the R/S statistics and plot it against the subsample length on double-logarithmic paper. The results of such an analysis for the first and last 660 returns are shown in Fig. 3. In both cases the slope of the empirical R/S statistics is similar -0.4282 and 0.4193, respectively. This confirms the stability of the $\mathrm{R} / \mathrm{S}$ analysis. The empirical slopes are also well below the theoretical, which for intervals in the range 10-330 is equal to 0.5779 .
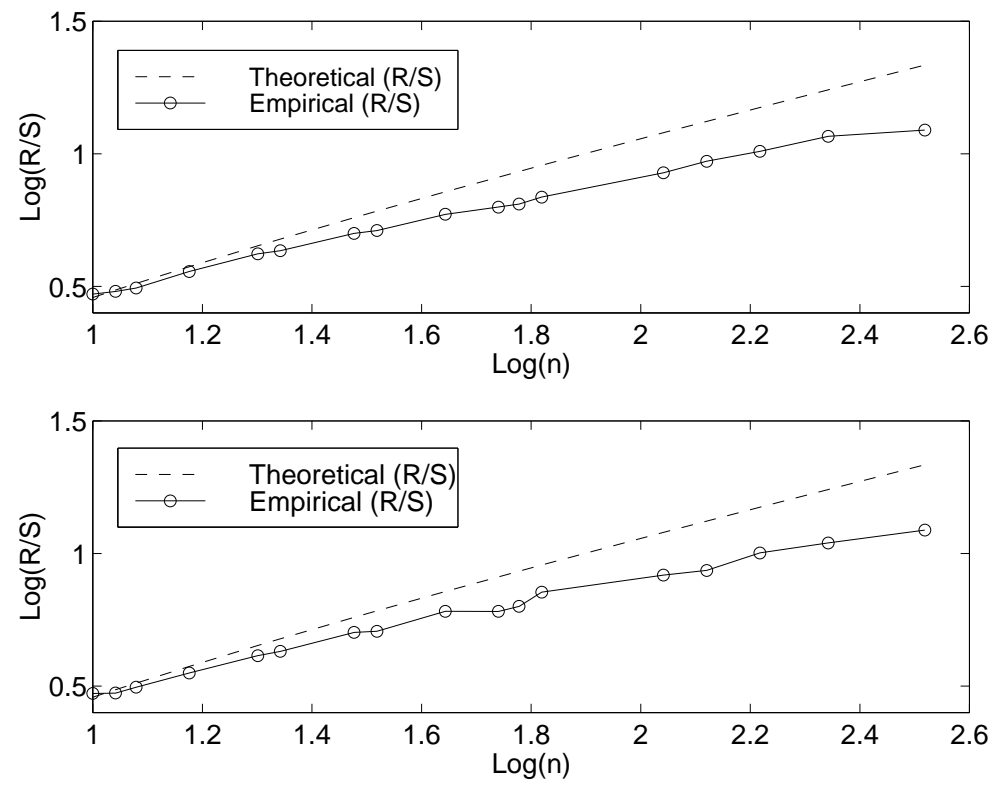

Figure 3: Hurst R/S analysis of the first (top) and last (bottom) 660 logarithmic returns of average daily prices from the CalPX spot market. The empirical Hurst exponents are 0.4282 and 0.4191 , respectively.

The second analyzed time series consists of the daily values of the Swiss Electricity Price (SWEP) index since March 11th, 1998 until March 31st, 2000. This gives us 525 data points, which are illustrated in the top panel of Fig. 4. All analysis is conducted on the 524 days long sequence of their logarithmic returns.

As before, in the first step of the Hurst analysis we have to find all divisors of the sample length. Since the number 524 has only two divisors greater or equal to 10 we have analyzed a sample of length 520, which has 10 divisors greater or equal to 10: $10,13,20,26,40,52,65,104,130$, and 260. Next we have calculated the R/S statistics and plotted it against the subsample length on double-logarithmic paper. The results for the first 520 returns (similar results were obtained for the last 520 returns) are presented in the top panel of Fig. 5. The slope of the empirical R/S statistics is 0.5291 , which is significantly below 0.5804 - the theoretical slope for subsamples in the range 10-260.

The SWEP index returns also possess the mean-reverting property, but only in the long run. Short term returns seem to be a completely random process. Why? A possible explanation is the calculation method of the index, which may somehow eliminate the short term dependence structure. The index is calculated for weekdays only, whereas CalPX prices are given for each day of the week (including Saturday 

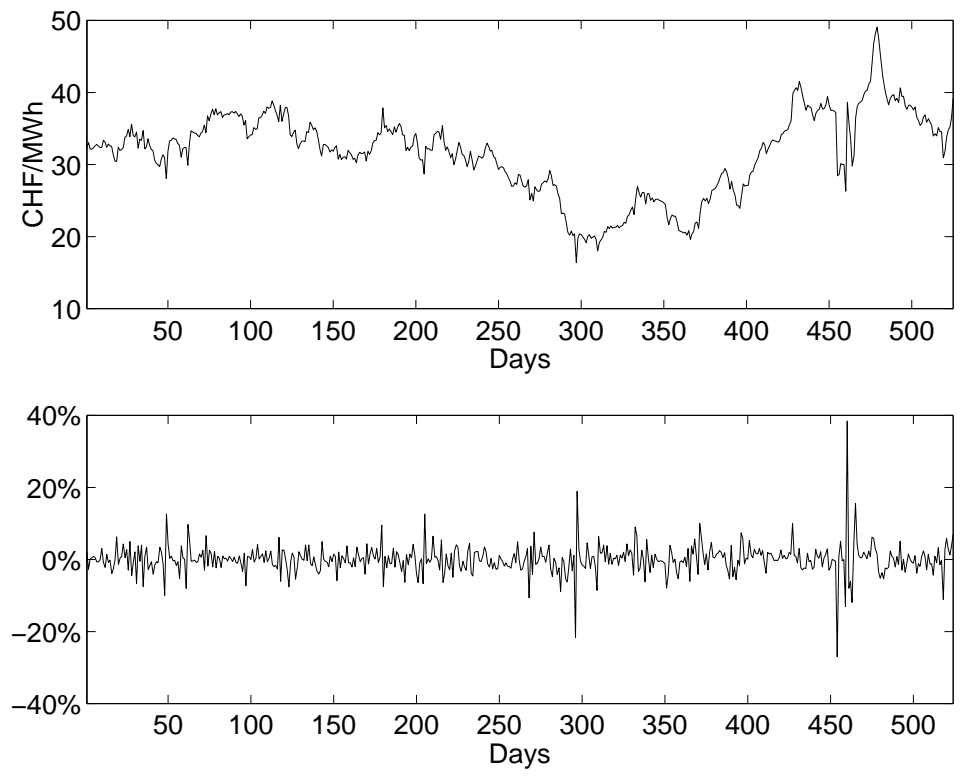

Figure 4: SWEP index since March 11th, 1998 until March 31st, 2000 (top) and logarithmic returns of the index (bottom).
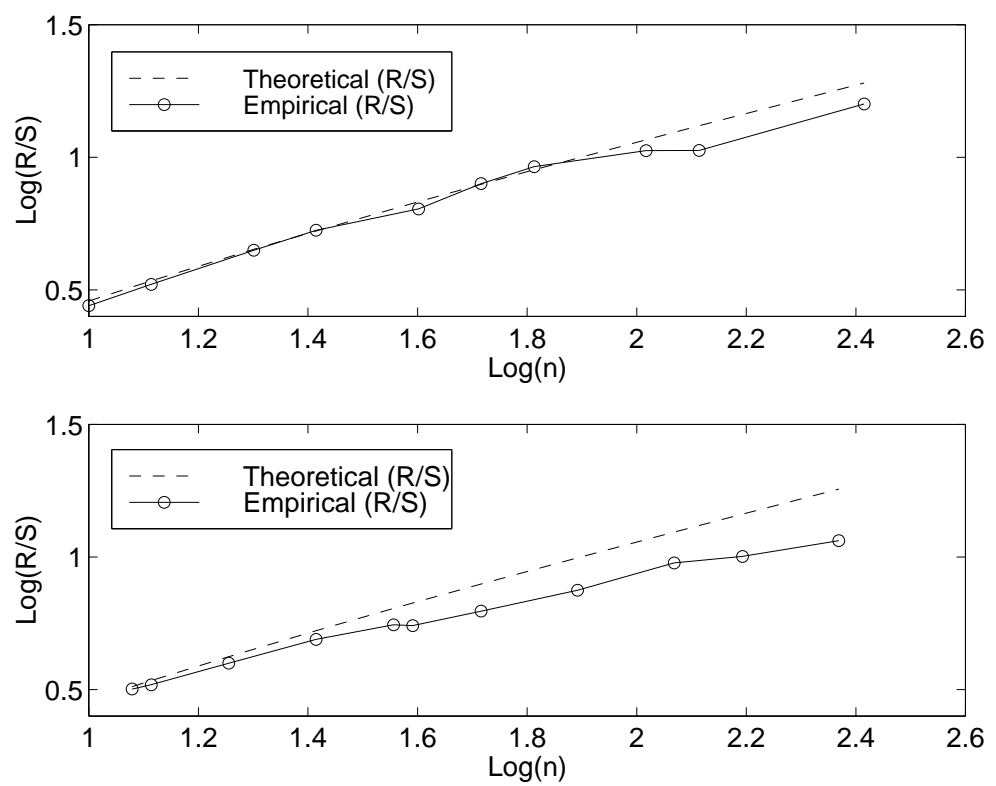

Figure 5: Hurst R/S analysis of the first 520 logarithmic returns of the SWEP index (top) and, for comparison, of the first 468 logarithmic returns of average weekday prices from the CalPX spot market (bottom). The empirical Hurst exponents are 0.5291 and 0.4391 , respectively. 
and Sunday). We checked if this is causing the difference by selecting only weekday average prices from the CalPX spot market and running the $\mathrm{R} / \mathrm{S}$ analysis for the obtained data set (478 returns). In the bottom panel of Fig. 5 we can clearly see that it is not. Anti-persistence of weekday returns is as strong as for all returns.

The next difference is the fact that SWEP is an indicator of the OTC electricity spot market prices (at the Swiss-German border) and not an exchange market clearing price. A complicated, six step procedure is used to derive the index value $[14,15]$. To calculate hourly prices from a given set of transactions, adjusted hourly prices for transactions longer than one hour have to be found. These hourly prices are based on price profiles, whereby the total turnover of the transaction remains unchanged. Unfortunately, we have not been able to check the impact of the calculation (averaging) procedure because the analyzed CalPX data set did not contain all needed information.

\section{$5 \quad$ Final remarks}

We have analyzed two data sets containing information about electricity prices in California and Central Europe. In both cases, the returns have been found to be mean-reverting processes. However, due to the calculation method of the SWEP index or, maybe, the difference between the exchange spot market in California and the Swiss-German OTC spot market the latter data set revealed a weaker antipersistence.

\section{References}

[1] J.P. Bouchaud, M. Potters, Theory of Financial Risk, (in French), Alea-Saclay, Eyrolles, Paris, 1997.

[2] R.N. Mantegna, H.E. Stanley, An Introduction to Econophysics: Correlations and Complexity in Finance, Cambridge University Press, Cambridge, 1999.

[3] A. Weron, R. Weron, Financial Engineering: Derivatives Pricing, Computer Simulations, Market Statistics, (in Polish), WNT, Warsaw, 1998.

[4] E.E. Peters, Fractal Market Analysis, Wiley, New York, 1994.

[5] M.M. Dacorogna, U.A. Müller, R.J. Nagler, R.B. Olsen, O.V. Pictet, J. Intern. Money Finance 12 (1993) 413.

[6] D.M. Guillaume, M.M. Dacorogna, R.R. Dave, U.A. Müller, R.B. Olsen, O.V. Pictet, Finance Stochast. 1 (1997) 95.

[7] R. Weron, Physica A (2000) in press.

[8] H.E. Hurst, Trans. Am. Soc. Civil Engineers 116 (1951) 770.

[9] B.B. Mandelbrot, J.R. Wallis, Water Resources Res. 5 (1969) 228.

[10] J. Beran, Statistics for Long-Memory Processes, Chapman \& Hall, New York, 1994.

[11] A.A. Anis, E.H. Lloyd, Biometrica 63 (1976) 283.

[12] International Chamber of Commerce, Liberalization and privatization of the Energy Sector, Paris, July 1998.

[13] S. Green, Power Engineering Int. 7(4) (1999) 45.

[14] See http://www.atel.ch/swep/.

[15] A. Weron, R. Weron, Power Exchange: Risk Management Strategies, (in Polish), CIRE, Wrocław, 2000. 


\section{HSC Research Report Series 2000}

For a complete list please visit http://ideas.repec.org/s/wuu/wpaper.html

01 Hurst analysis of electricity price dynamics by Rafał Weron and Beata Przybyłowicz 